\title{
Pretreatment neutrophil-to-lymphocyte ratio plus albumin-to-gamma-glutamyl transferase ratio predict the diagnosis of grade III glioma
}

\author{
Zhen-Qiang He, Hao Duan, Fu-Hua Lin, Ji Zhang, Yin-Sheng Chen, Guan-Hua Zhang, \\ Cheng-Cheng Guo, Chao Ke, Xiang-Heng Zhang, Zheng-He Chen, Jian Wang, Zhong-Ping Chen, \\ Xiao-Bing Jiang, Yong-Gao Mou \\ Department of Neurosurgery/Neuro-oncology, Sun Yat-sen University Cancer Center, State Key Laboratory of Oncology in South China, \\ Collaborative Innovation Center for Cancer Medicine, Guangzhou 510000, China \\ Contributions: (I) Conception and design: ZQ He, FH Lin, XB Jiang; (II) Administrative support: ZP Chen, XB Jiang, YG Mou; (III) Provision of \\ study materials or patients: FH Lin, J Zhang, YS Chen, GG Guo, C Ke, XH Zhang, ZH Chen, J Wang; (IV) Collection and assembly of data: ZQ \\ He, H Duan, GH Zhang, XB Jiang; (V) Data analysis and interpretation: ZQ He, H Duan, XB Jiang; (VI) Manuscript writing: All authors; (VII) \\ Final approval of manuscript: All authors. \\ Correspondence to: Yong-Gao Mou, MD, PhD; Xiao-Bing Jiang, MD, PhD. Department of Neurosurgery/Neuro-oncology, Sun Yat-sen University \\ Cancer Center, State Key Laboratory of Oncology in South China, Collaborative Innovation Center for Cancer Medicine, Guangzhou 510000, \\ China. Email: mouyg@sysucc.org.cn; jiangxiaob1@sysucc.org.cn.
}

Background: The present study explored the predictive value of systemic inflammatory indexes in diagnosing grade III gliomas of oligodendroglial origin.

Methods: A retrospective study of 154 patients with grade III gliomas was conducted. Systemic inflammatory indexes, including neutrophil-to-lymphocyte ratio (NLR), albumin-to-gamma-glutamyl transferase ratio (AGR), platelet-to-lymphocyte ratio, lymphocyte-to-monocyte ratio, prognostic nutritional index, and fibrinogen-to-albumin ratio, were reviewed. The resulting predictive model was externally validated using a demographic-matched cohort of 49 grade III glioma patients.

Results: In the training set, gliomas of oligodendroglial origin tended to have a lower NLR ( $\mathrm{P}=0.018)$ and a higher AGR $(\mathrm{P}=0.036)$ than those with tumors of astrocytic origin. Moreover, both NLR and AGR had predictive value for oligodendroglial tumors, when compared with astrocytic tumors. The best diagnostic value was obtained using NLR + AGR ( $\mathrm{AUC}=64.9 \%$, 95\% CI: 55.5-74.3\%, P=0.005). In the validation set, NLR + AGR satisfactorily predicted the presence of oligodendroglial tumors (AUC =66.5\%, 95\% CI: 50.6-82.4\%, $\mathrm{P}<0.05$ ) and co-deletion of $1 \mathrm{p} / 19 \mathrm{q}$ (AUC =73.7\%, 95\% CI: 59.2-88.1\%, P=0.005). Multivariate analysis further demonstrated NLR + AGR as an independent predictor for overall survival.

Conclusions: Pretreatment NLR and AGR aid in prognosis and diagnosing grade III oligodendroglial gliomas.

Keywords: Glioma; oligodendroglial; neutrophil-lymphocyte ratio (NLR); albumin-to-gamma-glutamyl transferase ratio (AGR); systemic inflammatory index

Submitted Jul 20, 2019. Accepted for publication Oct 24, 2019.

doi: $10.21037 /$ atm.2019.11.24

View this article at: http://dx.doi.org/10.21037/atm.2019.11.24 


\section{Introduction}

Gliomas are among the most common intracranial tumors. The majority are high grade gliomas with a very dismal prognosis, despite the application of optical treatments (1). A subgroup of patients with gliomas of oligodendroglial origin usually has much better survival $(1,2)$. Identifying these patients would allow clinicians to administer the most appropriate treatments. Generally, molecular markers, including co-deletion of $1 \mathrm{p} / 19 \mathrm{q}$ and IDH mutation, are involved in glioma differentiation (3). However, most gliomas are defined as post-operatively and are expensive to characterize. Glioma detection technologies remain unavailable in many medical centers globally. Therefore, identifying simple and non-invasive factors to predict gliomas of oligodendroglial origin preoperatively would be valuable.

Inflammation is a key component of the tumor microenvironment. Increasing evidence has highlighted the critical role of systemic inflammation in carcinogenesis, tumor metastasis, and tumor recurrence $(4,5)$. Systemic inflammatory indexes, including the neutrophil-tolymphocyte ratio (NLR) (6,7), platelet-to-lymphocyte ratio (PLR) (8), lymphocyte-to-monocyte ratio (LMR) (9), albumin-to-gamma-glutamyl transferase ratio (AGR) (10), prognostic nutritional index (PNI) (11), and fibrinogen-toalbumin ratio (FAR) (12) have been demonstrated to predict survival in various malignant tumors. The prognostic significance of these indexes for gliomas have also been investigated in previous studies, but mostly in glioblastomas (13-16). Moreover, the combination of NLR + LMR has been revealed as a non-invasive biomarker in glioblastoma diagnosis (17). Additionally, our previous studies have shown that preoperative PNI and a cumulative score based on albumin and fibrinogen levels predicted the overall survival (OS) of patients with high grade gliomas $(18,19)$. However, few studies have investigated the role of inflammatory indexes in grade III gliomas, which usually have a much better prognosis, especially those of oligodendroglial origin.

The loss of heterozygosity $(\mathrm{LOH})$ of $1 \mathrm{p} / 19 \mathrm{q}$ is the most important molecular aberration associated with oligodendrogliomas (20). Interestingly, a previous study has proposed that genes linked to inflammation are overexpressed in patients with intact $1 \mathrm{p} / 19 \mathrm{q}$ genes, explaining the more aggressive behavior of this subgroup of gliomas (21). Therefore, we hypothesized that the status of $1 \mathrm{p} / 19 \mathrm{q}$ co-deletion could be characterized by inflammatory indexes, thereby aiding in diagnosing gliomas of oligodendroglial origin.

To address this issue, we performed a retrospective study to assess the predictive roles of pretreatment levels of inflammatory indexes in the diagnosis of oligodendrogliomas and their prognostic significance for patients with grade III gliomas.

\section{Methods}

\section{Study population}

From January 2001 to December 2016, a total of 217 patients pathologically diagnosed with WHO grade III gliomas were retrospectively reviewed in our study. The following inclusion criteria were used: (I) pathological confirmation of WHO grade III gliomas, (II) absence of other malignancies, (III) absence of other anti-tumor treatments prior to admission, (IV) availability of complete clinical information and follow-up data. The exclusion criteria were as follows: (I) acute infection or chronic active inflammatory diseases, (II) autoimmune diseases or haematological disorders, (III) glucocorticoid treatment prior to admission and (IV) perioperative surgery-related mortality. and enrolled in the present study. Fourteen patients were excluded according to the criteria above. The remaining 203 patients were enrolled in this study. One hundred fifty-four patients treated from January 2001 to July 2014 were enrolled as the training set, and 49 patients identified from August 2014 to December 2016 were enrolled as the validation set (Figure S1). All the procedures in the current study were approved by the ethics committee of Sun Yat-sen University Cancer Centre. Written informed consent was obtained from all the patients.

\section{Data collection}

Demographic and clinical-pathological variables, including age, gender, Karnofsky performance status (KPS), pathological diagnoses, tumor size, tumor location, the extent of resection, and therapeutic information, were collected using an electronic medical record system. Formalin-fixed, paraffin-embedded tissue blocks, and slides were retrieved from the archives of the Pathology Department at Sun Yat-sen University Cancer Centre. The tumor size was defined as the maximum diameter measured on pretreatment enhanced T1-weighted MRI. The extent of resection was recorded as gross total resection (GTR), subtotal resection (STR), partial resection, and 
biopsy. The extent of resection was determined based on a contrast-enhanced head MRI scan performed within $72 \mathrm{~h}$ post-surgery, in addition to operation notes. Complete blood cell counts, serum albumin, and gamma-glutamyl transferase levels were evaluated before treatment. Systemic inflammatory indexes, including NLR, AGR, PLR, LMR, PNI, and FAR were reviewed.

For all the patients, follow-up started from the date of surgery. Patients were generally followed up every 3 months for the first year, semi-annually for the following 2 years, and annually thereafter. On follow-up, it was suggested that patients undergo a repeat contrasted MRI scan. By the last follow-up date, clinical attendance was verified through direct telecommunication with patients or their families. Overall survival (OS) was calculated from the day of surgery to the date of death or the date of the last follow-up visit.

\section{Evaluation of $\mathrm{O6}$ methylguanine-DNA-methyl transferase (MGMT) expression and $1 p / 19 q$ LOH status}

Surgical tissue specimens were routinely formalin-fixed, paraffin-embedded, and sectioned at a $5 \mu \mathrm{m}$ thickness for immunostaining using the streptavidin-biotin complex technique. All the slides were incubated with mouse monoclonal anti-MGMT at a 1:250 dilution (MT3.1; Abcam, Cambridge, UK). MGMT immunostaining was semi-quantitatively scored as $1=<5 \%, 2=5 \%$ to $10 \%, 3=10 \%$ to $25 \%$, and $4=>25 \%$, based on positive nuclear staining. A score of 1-2 was considered negative, whereas a score of 3-4 was positive.

The copy number statuses of $1 p$ and $19 q$ were assessed through fluorescence in situ hybridization (FISH) in formalin-fixed paraffin-embedded tumor tissues using a 1p36/1q25 Dual Color Probe, and a 19q13/19p13 Dual Color Probe (Vysis, Abbott Laboratories, Illinois, USA) in accordance with the manufacturer's instructions, with minor modifications. FISH signals for each locus-specific FISH probe were assessed using an Olympus BX51 TRF microscope (Olympus Corporation, Tokyo, Japan) equipped with a triple-pass filter (DAPI/Green/Orange, Vysis). At least 100 nonoverlapping nuclei were assessed to count a $1 \mathrm{p} / 1 \mathrm{q}$ ratio and a $19 \mathrm{q} / 19 \mathrm{p}$ ratio, and a ratio $<0.75$ was diagnosed as a loss. We try to obtain tumor tissue from all enrolled patients for $1 \mathrm{p} / 19 \mathrm{q} \mathrm{LOH}$ evaluation, but we failed to determine the $1 \mathrm{p} / 19 \mathrm{q} \mathrm{LOH}$ status of patients in the training set due to tissue quality. Thus, evaluation of $1 \mathrm{p} / 19 \mathrm{q}$ co-deletion was conducted only in the validation set.
Because of incomplete information concerning the status of $1 \mathrm{p} / 19 \mathrm{q}$, the diagnosis of grade III glioma was made in accordance with the 2007 WHO classification of central nervous system tumors (17).

\section{Statistical analysis}

Differences in baseline and clinicopathological parameters between groups were evaluated using a chi-square test or Fisher's exact test based on the type of data. All analyses were carried out using SPSS software, version 24.0 (IBM Corp., Armonk, NY, USA). The diagnostic performance of the systemic inflammatory indexes was assessed using values of the area under the curve (AUC) obtained from the receiver operating characteristic (ROC) curve. KaplanMeier survival analysis was performed. Differences between the curves were analyzed using the log-rank test. Cox proportional hazards models were adopted to calculate the hazard ratios (HRs) of death with regard to univariate or multivariate analysis. A two-tailed $\mathrm{P}$ value $<0.05$ was considered to be statistically significant.

\section{Results}

\section{Patient characteristics and treatment modality in the training set}

The baseline characteristics of all the patients in the training set are shown in Table 1. The median age of the patients was 40.0 years, and the majority of the patients were male $(\mathrm{n}=92,59.7 \%)$. A total of $114(74.0 \%)$ patients were diagnosed with anaplastic astrocytomas, $31(20.1 \%)$ with anaplastic oligodendrogliomas, and nine (5.8\%) with anaplastic oligoastrocytomas. The expression of MGMT was determined through immunohistochemistry (IHC) in $101(65.6 \%)$ patients, which was negative in 55 patients, and positive in 46 patients.

Samples used to assess the status of $1 \mathrm{p} / 19 \mathrm{q}$ deletion were only available in 27 patients, where the co-deletion of $1 \mathrm{p} / 19 \mathrm{q}$ was demonstrated in five patients. Additionally, single deletion at $1 \mathrm{p}$ and $19 \mathrm{q}$ was confirmed in one and six patients, respectively. GTR was achieved in more than half of all patients $(n=90,58.4 \%)$, and $29.9 \%(n=46)$ of patients underwent STR, while $11.7 \%$ underwent partial resection ( $n=19)$. Adjuvant radiotherapy and chemotherapy were undertaken in 84 patients $(54.5 \%)$. The treatment modalities for all the patients in the training set are summarized in Table 2. 


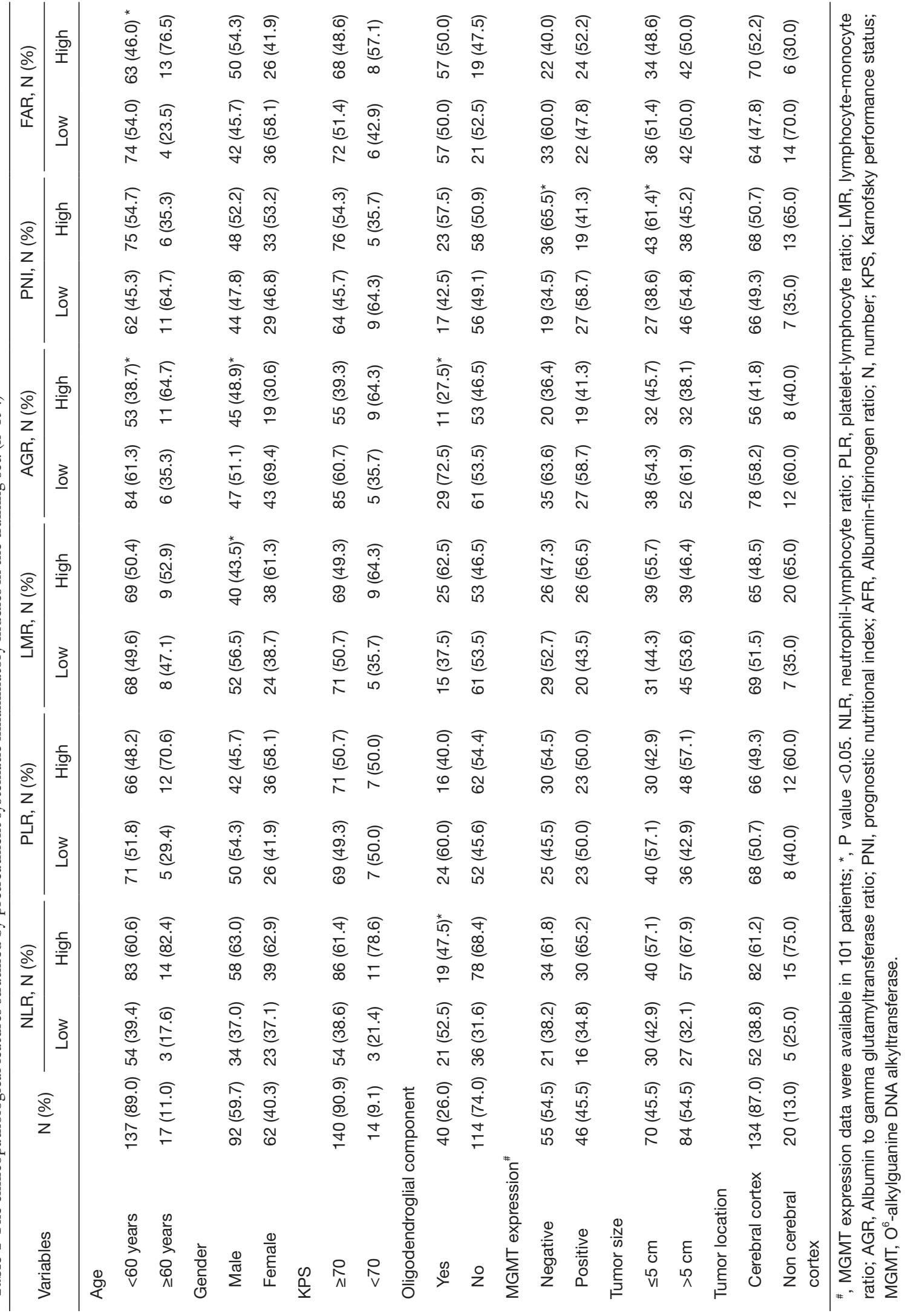


Table 2 Treatment modality for the patients in training set $(n=154)$

\begin{tabular}{lc}
\hline Treatment madality & $\mathrm{N}(\%)$ \\
\hline Surgical resection only & $42(27.3)$ \\
Surgery $\rightarrow$ Radiotherary & $21(13.6)$ \\
Surgery $\rightarrow$ Radiotherary $\rightarrow$ Chemotheraphy $(\mathrm{A})$ & $55(35.7)$ \\
Surgery $\rightarrow$ Radiotherary $\rightarrow$ Chemotheraphy $(\mathrm{B})$ & $29(18.8)$ \\
Surgery $\rightarrow$ Chemotheraphy $(\mathrm{A})$ & $2(1.3)$ \\
Surgery $\rightarrow$ Chemotheraphy $(\mathrm{B})$ & $5(3.2)$
\end{tabular}

A, Temozolomide-based; B, nitrosourea-based or platinumbased.

Table 3 Preoperative value of systematic inflammation markers

\begin{tabular}{lcc}
\hline Indexes & Median (range) & Cut off value \\
\hline NLR & $2.871(0.667-22.43)$ & 2.295 \\
PLR & $123.3(35.58-531.4)$ & 123.3 \\
LMR & $4.333(0.500-27.00)$ & 4.333 \\
AGR & $1.877(0.147-9.756)$ & 1.712 \\
PNI & $52.95(40.40-70.89)$ & 52.55 \\
FAR & $0.0594(0.0348-0.1530)$ & 0.0597 \\
\hline
\end{tabular}

NLR, neutrophil-to-lymphocyte ratio; PLR, platelet-tolymphocyte ratio; LMR, lymphocyte-to-monocyte ratio; AGR, albumin-to-gamma-glutamyl transferase ratio; PNI, prognostic nutritional index; FAR, fibrinogen-to-albumin ratio.

\section{NLR and AGR were significantly correlated with tumor oligodendroglial components}

The median values and ranges of the inflammation indexes are presented in Table 3. The optimal cut-offs for NLR, PNI, AGR, and FAR were determined through ROC analysis (Figure 1). The median value was set as the cut-off for LMR and PLR since there were no significant results in the ROC analysis. To explore the potential associations between clinical-pathological features and inflammation indexes, patients were divided into two groups based on the cut-off of each index (Table 1).

Intriguingly, patients with gliomas containing an oligodendroglial component were inclined to have lower NLR $(\mathrm{P}=0.018)$ and higher AGR $(\mathrm{P}=0.036)$, than those with tumors of astrocytic origin. In contrast, the other four indexes were not found to be associated with the presence of an oligodendroglial component. PNI was associated with tumor size and MGMT expression levels. In addition, as shown in Table 1, LMR, and AGR were significantly associated with gender, while FAR and AGR were associated with age (all $\mathrm{P}<0.05)$.

Moreover, we assessed the association between the combination of NLR + AGR and the presence of an oligodendroglial component. According to the optimal cutoff values of NLR and AGR, patients with NLR $\leq 2.295$ were attributed with a score of 0 , and patients with NLR $>2.295$ were attributed with a score of 1 . Patients with AGR $\geq 1.712$ were attributed with a score of 0 , and patients with AGR $<1.712$ were attributed with a score of 1 . After the combination of NLR and AGR scores, patients with elevated NLR and decreased AGR levels were allocated to a score $=2$, those with only elevated NLR or only decreased AGR were allocated to a score $=1$ and those with decreased NLR and elevated AGR levels were allocated with a score $=0$. Based on the accumulative score of NLR + AGR, Patients were divided into three subgroups. NLR + AGR was demonstrated to be strongly negatively correlated with the presence of an oligodendroglial component $\left(\chi^{2}=9.78\right.$, $\mathrm{P}=0.008$ ).

\section{Systemic inflammatory indexes predict prognosis in $\mathrm{WHO}$ grade III gliomas}

To investigate the prognostic potential of these systemic inflammatory indexes, patients in the training set were divided into two groups in accordance with the cut-off values determined through ROC analysis or median values. The median survival of patients in the training set was 25.53 months. As shown in Figure 2, only LMR failed to show any prognostic power, while NLR $(\mathrm{P}=0.002)$, PLR $(\mathrm{P}=0.021)$, AGR $(\mathrm{P}<0.001), \mathrm{PNI}(\mathrm{P}<0.001)$ and FAR $(\mathrm{P}<0.001)$ can significantly predict $\mathrm{OS}$ in grade III glioma patients.

\section{$N L R+A G R$ aids in diagnosing oligodendroglial tumors and predicts chromosomal deletion at $1 p / 19 q$}

Next, we further assessed the efficacy of NLR and AGR in predicting the presence of an oligodendroglial component. A ROC analysis indicated that both NLR (AUC $=62.6 \%$, 95\% CI: $53.1-72.1 \%, \mathrm{P}=0.018$ ) and AGR (AUC $=60.5 \%$, 95\% CI: $50.0-71.0 \%, \mathrm{P}=0.048)$ had significant predictive value for tumors of oligodendroglial origin, when compared with those of astrocytic origin. The best diagnostic value was obtained using the combination of NLR + AGR (AUC 

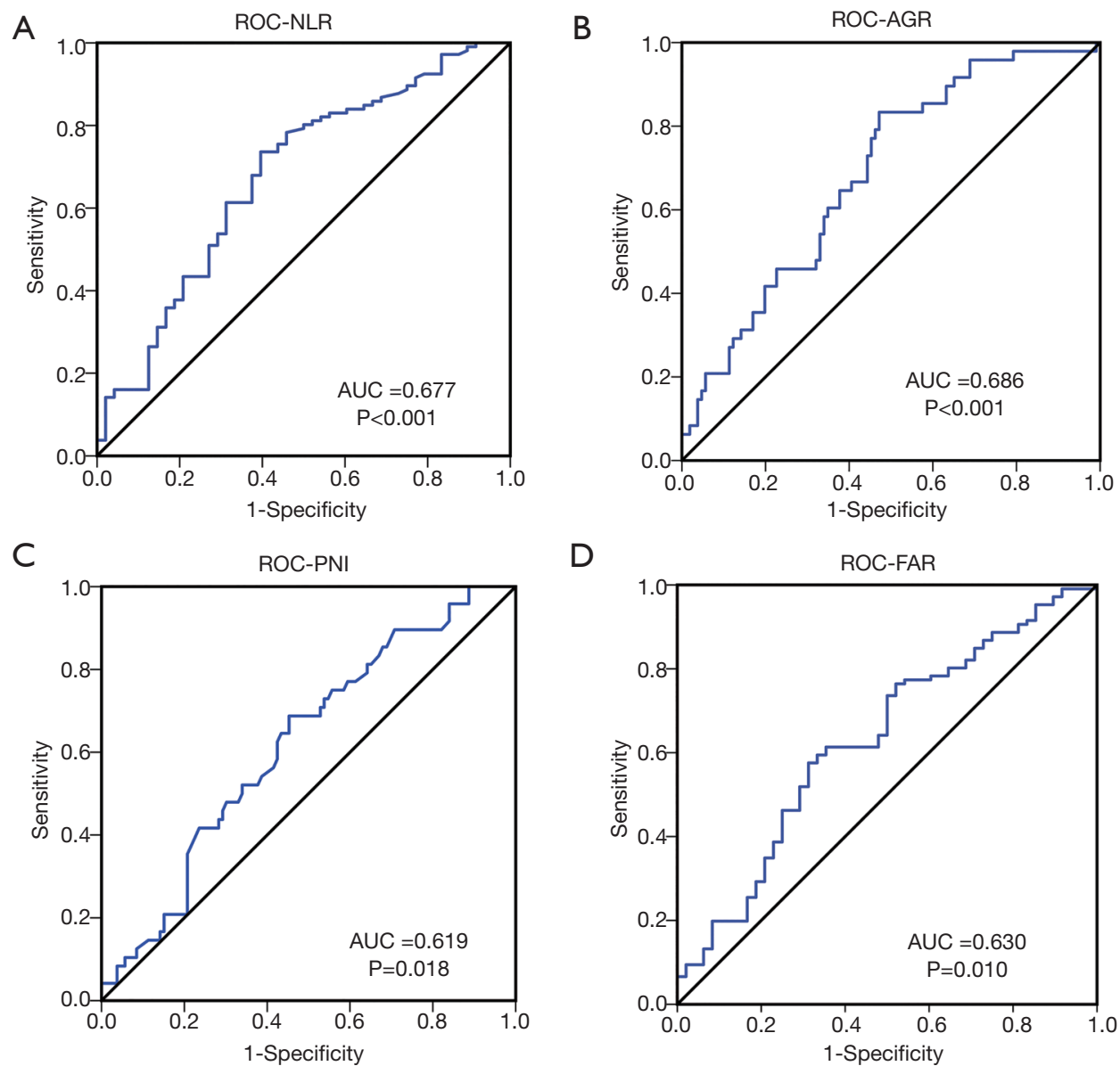

Figure 1 ROC curves. Receiver operating characteristic (ROC) analyses were performed to evaluate the optimal cut-off values of systemic inflammatory indexes. NLR (A), AGR (B), PNI (C) and FAR (D) showed statistically significant results, and cut-off values were determined using the Youden index. Since PLR and LMR failed in the ROC analysis, median values were set as the cut-off value. NLR, neutrophil-tolymphocyte ratio; AGR, albumin-to-gamma-glutamyl transferase ratio; PNI, prognostic nutritional index; FAR, fibrinogen-to-albumin ratio; PLR, platelet-to-lymphocyte ratio; LMR, lymphocyte-to-monocyte ratio.

$=64.9 \%$, 95\% CI: $55.5-74.3 \%, \mathrm{P}=0.005$ ) (Figure $3 A$ ). Furthermore, the combination score based on NLR and AGR levels showed significant prognostic power in $\mathrm{WHO}$ grade III glioma patients $(\mathrm{P}<0.001)$ (Figure $3 B)$.

An independent set of 49 consecutive patients with grade III gliomas was used to validate the predictive capability of NLR + AGR statistically. There was no significant difference between the training and validation datasets with regard to age, KPS, the extent of resection, and treatment modalities (all $\mathrm{P}>0.05$ ). A ROC analysis indicated that NLR + AGR had significant predictive value for the presence of an oligodendroglial component (AUC $=66.5 \%, 95 \%$ CI: $50.6-82.4 \%, \mathrm{P}<0.05)$ (Figure $4 A$ ).

Furthermore, considering the key role of $1 \mathrm{p} / 19 \mathrm{q} L O H$ in the diagnosis of oligodendrogliomas, we also evaluated the efficacy of NLR + AGR in predicting the co-deletion of $1 \mathrm{p} / 19 \mathrm{q}$, compared with a single deletion or intact $1 \mathrm{p} / 19 \mathrm{q}$ in the validation set. NLR + AGR displayed a satisfactory predictive capability (AUC $=73.7 \%, 95 \%$ CI: $59.2-88.1 \%$, $\mathrm{P}=0.005$ ) (Figure 4B).

\section{Univariate and multivariate Cox regression analysis of the role of $N L R+A G R$ in predicting $O S$}

In the training set, the median duration of follow-up was 22.0 months (interquartile range, $14.2-45.0$ months). At the last follow-up, 106 (68.8\%) patients had died, with a median OS of 25.53 months (95\% CI: 16.90-34.16 months). Age, 
A

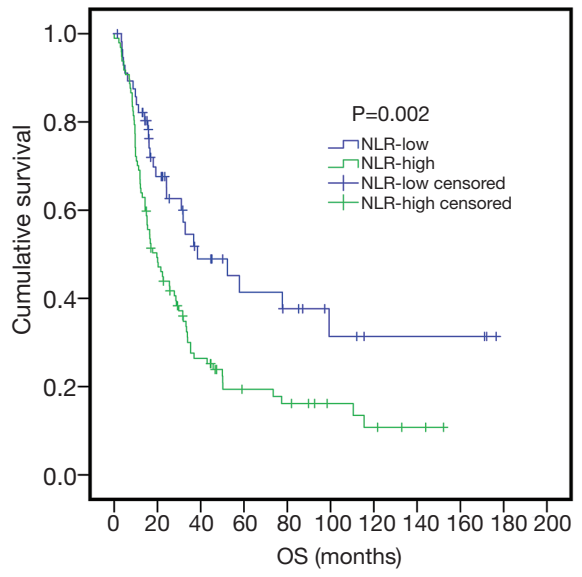

D

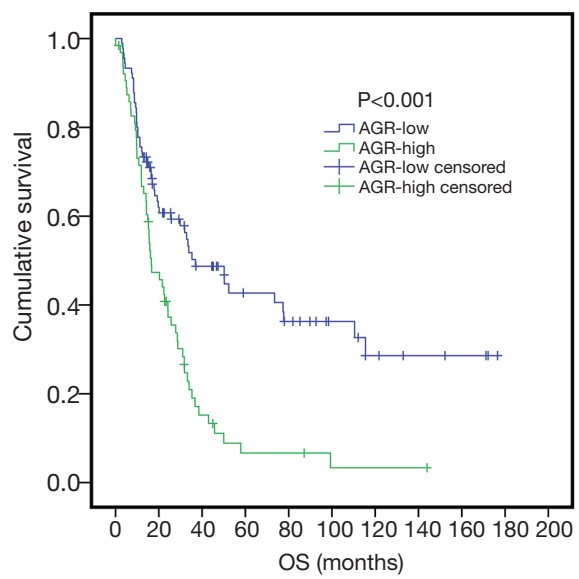

B

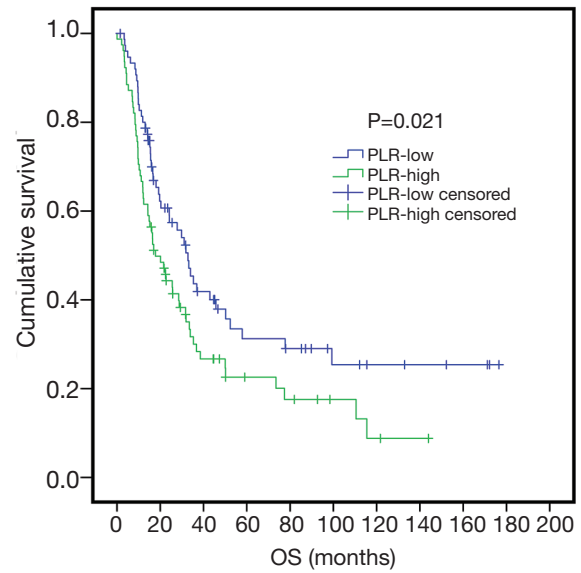

$\mathrm{E}$

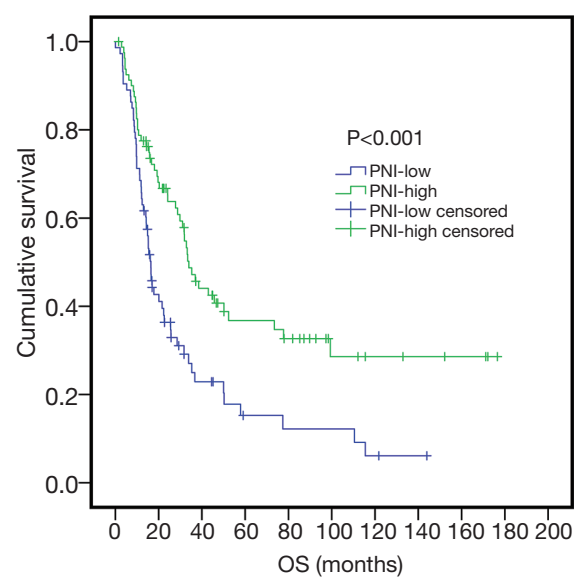

C

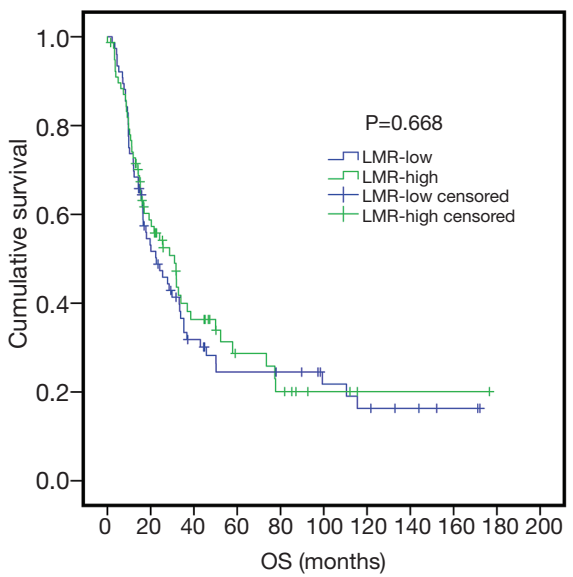

$\mathrm{F}$

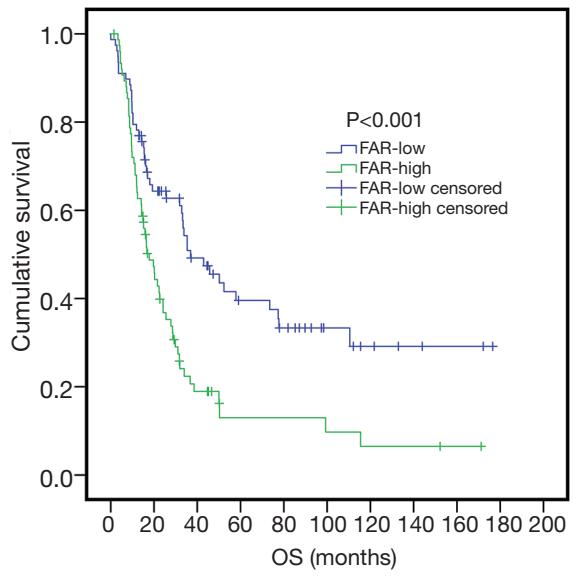

Figure 2 Kaplan-Meier survival curves of WHO grade III glioma patients in the training set. Patients were divided into two groups according to the cut-off values of all six inflammatory indexes. Cut-off values of all six indexes are shown in Table 3. Five out of six inflammatory indexes, including NLR ( $\mathrm{A}, \mathrm{P}=0.002)$, PLR ( $\mathrm{B}, \mathrm{P}=0.021)$, AGR ( $\mathrm{D}, \mathrm{P}<0.001)$, PNI $(\mathrm{E}, \mathrm{P}<0.001)$ and FAR $(\mathrm{F}, \mathrm{P}<0.001)$ can significantly predict $\mathrm{OS}$ in grade III glioma patients. $\mathrm{LMR}(\mathrm{C}, \mathrm{P}=0.668)$ fails to show significant result in survival analysis. NLR, neutrophilto-lymphocyte ratio; AGR, albumin-to-gamma-glutamyl transferase ratio; PNI, prognostic nutritional index; FAR, fibrinogen-to-albumin ratio; PLR, platelet-to-lymphocyte ratio; LMR, lymphocyte-to-monocyte ratio.

gender, KPS, the extension of resection, tumor location, treatment modality, MGMT expression, and NLR + AGR were included in the Cox regression analysis.

In the univariate analysis, NLR + AGR, along with traditional predictors, including age, KPS, the extent of resection, and adjuvant chemo-radiotherapy, showed significant associations with $\mathrm{OS}($ all $\mathrm{P}<0.05)$. In a multivariate model, NLR + AGR remained a risk factor for OS independent of the other factors (Table 4). Patients with an NLR + AGR value of 2 had a 3.9-fold increased risk of death compared with those with an NLR + AGR of 0 .

\section{Discussion}

High grade gliomas are usually associated with very poor prognosis. However, a subgroup of patients with an oligodendroglial component has much better survival (2). The presence of a $1 \mathrm{p} / 19 \mathrm{q}$ co-deletion is essential in diagnosing oligodendrogliomas, but this can only be assessed postoperatively and is expensive to measure. Therefore, it is of value to explore noninvasive and economical methods in predicting gliomas of oligodendroglial origin.

Our study showed that only NLR and AGR 

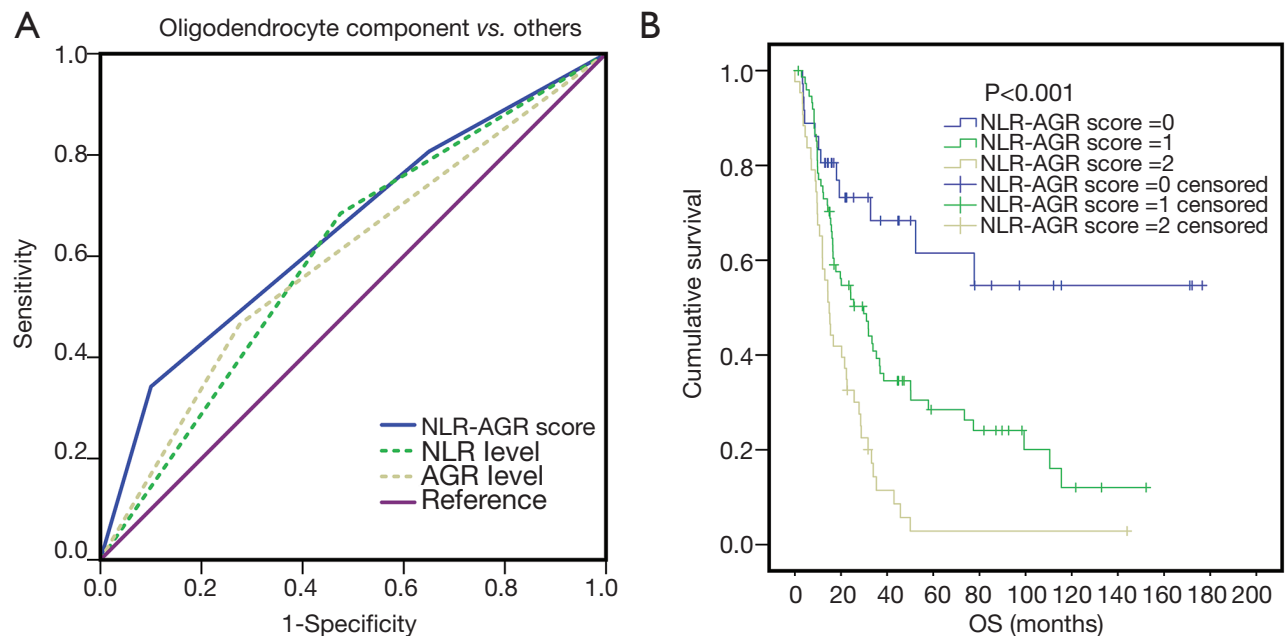

Figure 3 Predictive value of NLR-AGR score for WHO grade III glioma patients in the training set. (A) ROC analysis showed that the combined NLR-AGR score had a larger AUC (AUC =64.9\%, 95\% CI: 55.5-74.3\%, P=0.005) than that of NLR (AUC =62.6\%, 95\% CI: 53.1-72.1\%, P=0.018) or AGR (AUC =60.5\%, 95\% CI: 50.0-71.0\%, P=0.048) alone in predicting an oligodendroglial component in grade III glioma patients. (B) The Kaplan-Meier method and a log-rank test were used to investigate the differences in OS in grade III glioma patients by NLR-AGR score. Patients with a low NLR-AGR score demonstrated a higher OS compared with patients with a higher NLRAGR score $(\mathrm{P}<0.001)$. NLR, neutrophil-to-lymphocyte ratio; AGR, albumin-to-gamma-glutamyl transferase ratio.
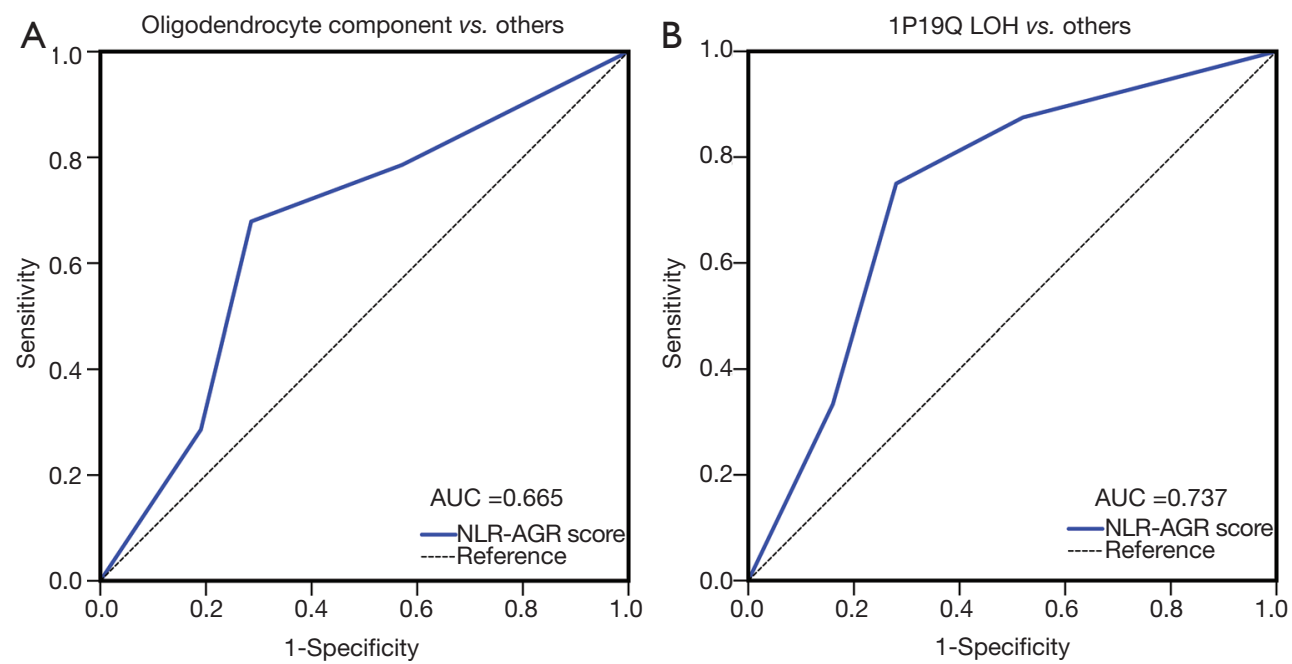

Figure 4 Predictive value of NLR-AGR score for WHO grade III glioma patients in the validation set. (A) ROC analysis showed that the NLR-AGR score can significantly predict the oligodendrocyte component in the validation set (AUC =66.5\%, 95\% CI: 50.6-82.4\%, $\mathrm{P}<0.05)$. (B) ROC analysis showed that the NLR-AGR score can also significantly predict $1 \mathrm{p} / 19 \mathrm{q}$ LOH in the validation set $(\mathrm{AUC}=73.7 \%$, 95\% CI: 59.2-88.1\%, P=0.005). NLR, neutrophil-to-lymphocyte ratio; AGR, albumin-to-gamma-glutamyl transferase ratio. 
Table 4 The multivariate analysis of the prognostic factors for OS in patients with WHO Grade III gliomas in the training set (n=154)

\begin{tabular}{|c|c|c|c|}
\hline Variables & \multicolumn{3}{|c|}{ Overall survival } \\
\hline Age, years & & & 0.003 \\
\hline$<60$ & 1 & Reference & \\
\hline$\geq 60$ & 2.49 & $1.37-4.53$ & \\
\hline$\geq 70$ & 1 & Reference & \\
\hline$<70$ & 1.99 & $1.06-3.75$ & \\
\hline \multicolumn{4}{|l|}{ Extent of resection } \\
\hline Gross total resection & 1 & Reference & \\
\hline $\begin{array}{l}\text { Adjuvant radiotherapy and } \\
\text { chemotherapy }\end{array}$ & & & $<0.001$ \\
\hline Yes & 1 & Reference & \\
\hline No & 2.40 & $1.61-3.58$ & \\
\hline \multicolumn{4}{|l|}{ NLR-AGR Score } \\
\hline 0 & 1 & Reference & $<0.001$ \\
\hline 1 & 2.34 & $1.22-4.56$ & 0.010 \\
\hline 2 & 3.88 & $1.97-7.67$ & $<0.001$ \\
\hline
\end{tabular}

HR, hazard ratio; Cl, confidence interval; KPS, Karnofsky performance status; NLR, neutrophil-lymphocyte ratio; AGR, albumin-gammaglutamyltransferase ratio.

were significantly associated with the presence of an oligodendroglial component. The combination of NLR + AGR has a satisfactory predictive value in the diagnosis of oligodendrogliomas and the co-deletion of $1 \mathrm{p} / 19 \mathrm{q}$. Furthermore, patients with NLR $>2.295$ and AGR $<1.712$ had a 3.9 -fold increased risk of death compared with those with NLR $\leq 2.295$ and AGR $\geq 1.712$. To the best of our knowledge, our study is the first to reveal the predictive value of inflammatory indexes in the diagnosis of oligodendrogliomas.

The current study revealed that NLR and AGR could serve as economical and non-invasive markers predicting the presence of an oligodendroglial component among patients with grade III gliomas. Moreover, a combination of NLR and AGR also had a strong independent association with the OS of patients with grade III gliomas. Although FISH is a routine method to determine $1 \mathrm{p} / 19 \mathrm{q}$ status, this method remains unavailable in some institutes. Moreover, FISH false-positive results are another problem compromising the application and reliability of $1 \mathrm{p} 19 \mathrm{q}$ status detection. Therefore, the value of NLR and AGR in predicting the presence of an oligodendroglial component and OS could provide an alternative to direct $1 \mathrm{p} 19 \mathrm{q}$ status detection results and could help in evaluating patient prognosis and planning treatment before surgery.

Generally, glioma patients with an oligodendroglial component have much better survival than those without. According to updated classifications, the diagnosis of an anaplastic oligodendroglioma requires the presence of $1 \mathrm{p} / 19 \mathrm{q}$ co-deletion (3). In the present study, the majority of patients in the training set lacked information concerning their $1 \mathrm{p} / 19 \mathrm{q}$ status, since all of them were treated before August 2014. We tried to obtain this information but failed. To address this issue, we enrolled a validation set of patients treated post-August 2014, where $1 \mathrm{p} / 19 \mathrm{q}$ status was available. The association between the presence of 
an oligodendroglial component and NLR plus AGR was confirmed in the validation set. Moreover, lower NLR and higher AGR also significantly predicted the co-deletion of $1 \mathrm{p} / 19 \mathrm{q}$, which is a strong predictor of prolonged survival. Previous studies have demonstrated that both NLR and AGR could either reflect the level of systemic inflammation or immune proliferation $(10,13)$. Genes linked to the immune response and inflammation were shown to be overexpressed in oligodendroglial tumors with intact $1 \mathrm{q} 19 \mathrm{q}(21)$. These findings could help to explain the predictive significance of NLR and AGR in the diagnosis and prognosis of oligodendroglial tumors. However, the causative relationship between these factors is currently questionable, and potential mechanisms deserve further investigation.

Cancer can evoke local and systemic inflammatory responses, which have been recognized as cancer-related inflammation, a hallmark feature of cancer development and progression (22). The brain was considered to be immune-privileged, although this concept has been redefined recently since functional lymphatic vessels carrying both fluid and immune cells from the cerebrospinal fluid were observed in the dural sinuses (23). Recently, Chongsathidkiet et al. reported that sequestration of $\mathrm{T}$ cells in bone marrow, which was mediated by the loss of surface sphingosine-1-phosphate receptor 1 on $\mathrm{T}$ cells, was only observed in intracranial tumors (24). Jackson et al. also reported that the systemic deletion and systemically impaired cytotoxicity of antigen-specific CD8+ T cells were more obvious in intracranial melanoma than in equivalently progressed extracranial tumors (25). These studies suggest that brain tumors might also influence systemic immune function. Previously, several systemic inflammatory indexes, including NLR, PLR, LMR, AGR, PNI, and FAR have been demonstrated to predict survival in various malignant tumors (6-12). Similarly, the role of these indexes has also been explored in glioblastomas (13-16). In the present study, we investigated their prognostic significance in WHO grade III gliomas. In line with results in glioblastoma patients, NLR, PLR, PNI, and FAR were shown to predict OS in grade III glioma patients.

We also evaluated the role of AGR in grade III glioma and found that AGR was also an independent prognostic factor. Moreover, the combination of AGR and NLR was demonstrated to be the most powerful predictor of OS. AGR was reported to serve as an independent favorable prognostic factor in intrahepatic cholangiocarcinoma patients (10). However, its prognostic value has not been previously determined in gliomas. The mechanism underlying the association between AGR and patient outcomes is not very clear. Generally, reduced albumin levels and elevated gamma-glutamyl transferase (GGT) imply impaired liver function and poor nutritional status, which have been widely acknowledged as predictors of poor outcomes in cancer patients. In addition, oxidative stress, a major product of inflammation that plays an important role in carcinogenesis (26), may also partially account for this association between AGR and patient outcomes (10). Physically, albumin provides abundant antioxidative substances (27). However, GGT plays a key role in the metabolism of intracellular glutathione, which protects the cell from oxidative damage (28). Therefore, AGR is more than a proxy of nutritional status and liver function but is also a systemic inflammatory index indicating antioxidant balance.

Generally, an increased NLR represents either a decrease in the total lymphocyte count and/or an increase in the total neutrophil count. The lymphocyte count is an index of cell-mediated immunity and plays a central role in the cytotoxic immune response of the host. Critical subpopulations of lymphocytes, such as CD4+ T helper type 1 lymphocytes, CD8+ cytotoxic T lymphocytes, and natural killer $\mathrm{T}$ cells, have multiple anticancer faculties. They can detect and eliminate pre-malignant and malignant tumor cells indirectly or directly. These cells can also prevent angiogenesis and metastasis (29). Decreased lymphocyte counts are associated with impaired cellular immunity, resulting in tumor progression and poor survival. A low level of CD8+ tumor-infiltrating lymphocytes is reported to be associated with an unfavorable prognosis in glioblastoma (30), while the presence of neutrophils in the tumor microenvironment has been correlated with poor prognosis in several studies (31). Neutrophils are the first line of defense during inflammation and infections. Neutrophils have antimicrobial functions such as phagocytosis or cytokine and chemokine production (32). However, neutrophils are also found to infiltrate in many types of tumor and tumor-associated neutrophils (TANs) are reported to be involved in genotoxicity, angiogenesis, and immunosuppression (33). TANs may exert pro-tumoral functions by favoring genetic instability through the release of reactive oxygen species, promoting tumor cell proliferation through elastase, sustaining angiogenesis through the release of vascular 
endothelial growth factor or matrix metalloproteases-9, enhancing cancer cell invasiveness through soluble mediators such as hepatocyte growth factor, and suppressing effective CD8+ T-cell immunity through the expression of arginase $(34,35)$. Therefore, a higher level of NLR in glioma patients indicates a decrease in lymphocytes and/or an increase in neutrophils, which may also indicate impaired lymphocyte immunological responses and/or increased pro-tumoral TANs. This may explain why a higher NLR indicates poorer survival.

As with most retrospective studies, our study has several limitations. Firstly, this is a retrospective analysis with a moderate sample size and a single-center design, which may be subject to selection bias. Secondly, we failed to obtain integrated molecular mutation data, including isocitrate dehydrogenase 1/2 (IDH1/2) mutation, methylation status of telomerase reverse transcriptase (TERT) promoter, and $\alpha$-thalassemia/mental retardation syndrome $\mathrm{X}$-linked (ATRX) mutation, which are known to be prognostic factors for glioma patients. Despite its preliminary nature, the current study has clearly and convincingly showed the prognostic value and predictive power of the NLRAGR score for grade III glioma patients. However, more prospectively designed trials are needed to validate our findings.

In summary, the present study showed that a combination of pretreatment NLR and AGR could serve as a valuable factor for predicting the presence of an oligodendroglial component and better OS in WHO grade III glioma patients. Both NLR and AGR are easily accessible, noninvasive, and inexpensive markers that could help in guiding the individualized treatment of grade III glioma patients.

\section{Acknowledgments}

We thank Conn Hastings, PhD, from Liwen Bianji, Edanz Editing China (www.liwenbianji.cn/ac), for editing the English text of a draft of this manuscript. We thank AME Editing Service (http://editing.amegroups.com/) for editing the proper English language, grammar, punctuation, spelling and overall style of this manuscript.

Funding: This work was supported by Science and Technology Planning Project of Guangdong Province, China [grant number 2013B090500095], [grant number 2014A020212576], [grant number 2015B010125003]; Science and Technology Program of Guangzhou, China [grant number 201704020133]; National Natural Science foundation of China [grant number 81872324]. The funders had no role in the study design, data collection and analysis, decision to publish, or preparation of the manuscript.

\section{Footnote}

Conflicts of Interest: The authors have no conflicts of interest to declare.

Ethical Statement: The authors are accountable for all aspects of the work in ensuring that questions related to the accuracy or integrity of any part of the work are appropriately investigated and resolved. All procedures performed in studies involving human participants were in accordance with the ethical standards of the institutional and/or national research committee and with the 1964 Helsinki declaration and its later amendments or comparable ethical standards. This study was approved by the Institutional Review Board of Sun Yat-Sen University Cancer Center (No. GZR2018-244). The raw data in this paper has been successfully uploaded and locked onto Research Data Deposit with a RDD number of RDDA2019000999. All patients have provided written informed consent for their clinical information to be used in our study.

\section{References}

1. Ostrom QT, Gittleman H, Truitt G, et al. CBTRUS Statistical Report: Primary Brain and Other Central Nervous System Tumors Diagnosed in the United States in 2011-2015. Neuro Oncol 2018;20:iv1-iv86.

2. Donahue B, Scott CB, Nelson JS, et al. Influence of an oligodendroglial component on the survival of patients with anaplastic astrocytomas: A report of radiation therapy oncology group 83-02. Int J Radiat Oncol Biol Phys 1997;38:911-4.

3. Louis DN, Perry A, Reifenberger G, et al. The 2016 World Health Organization Classification of Tumors of the Central Nervous System: a summary. Acta Neuropathol 2016;131:803-20.

4. Crusz SM, Balkwill FR. Inflammation and cancer: advances and new agents. Nat Rev Clin Oncol 2015;12:584-96.

5. Diakos CI, Charles KA, McMillan DC, et al. Cancerrelated inflammation and treatment effectiveness. Lancet Oncol 2014;15:e493-503.

6. Gu XB, Tian T, Tian XJ, et al. Prognostic significance 
of neutrophil-to-lymphocyte ratio in non-small cell lung cancer: a meta-analysis. Sci Rep 2015;5:12493.

7. Koh CH, Bhoo-Pathy N, Ng KL, et al. Utility of pretreatment neutrophil-lymphocyte ratio and plateletlymphocyte ratio as prognostic factors in breast cancer. $\mathrm{Br}$ J Cancer 2015;113:150-8.

8. Kim EY, Lee JW, Yoo HM, et al. The Platelet-toLymphocyte Ratio Versus Neutrophil-to-Lymphocyte Ratio: Which is Better as a Prognostic Factor in Gastric Cancer? Ann Surg Oncol 2015;22:4363-70.

9. Stotz M, Pichler M, Absenger G, et al. The preoperative lymphocyte to monocyte ratio predicts clinical outcome in patients with stage III colon cancer. Br J Cancer 2014;110:435-40.

10. Jing CY, Fu YP, Shen HJ, et al. Albumin to gammaglutamyltransferase ratio as a prognostic indicator in intrahepatic cholangiocarcinoma after curative resection. Oncotarget 2017;8:13293-303.

11. Chen KL, Liu YH, Li WY, et al. The prognostic nutritional index predicts survival for patients with extranodal natural killer/T cell lymphoma, nasal type. Ann Hematol 2015;94:1389-400.

12. Tan Z, Zhang M, Han Q, et al. A novel blood tool of cancer prognosis in esophageal squamous cell carcinoma: the Fibrinogen/Albumin Ratio. J Cancer 2017;8:1025-9.

13. Lopes M, Carvalho B, Vaz R, et al. Influence of neutrophillymphocyte ratio in prognosis of glioblastoma multiforme. J Neurooncol 2018;136:173-80.

14. Bambury RM, Teo MY, Power DG, et al. The association of pre-treatment neutrophil to lymphocyte ratio with overall survival in patients with glioblastoma multiforme. J Neurooncol 2013;114:149-54.

15. Han S, Liu Y, Li Q, et al. Pre-treatment neutrophil-tolymphocyte ratio is associated with neutrophil and T-cell infiltration and predicts clinical outcome in patients with glioblastoma. BMC Cancer 2015;15:617.

16. McNamara MG, Lwin Z, Jiang H, et al. Factors impacting survival following second surgery in patients with glioblastoma in the temozolomide treatment era, incorporating neutrophil/lymphocyte ratio and time to first progression. J Neurooncol 2014;117:147-52.

17. Zheng SH, Huang JL, Chen M, et al. Diagnostic value of preoperative inflammatory markers in patients with glioma: a multicenter cohort study. J Neurosurg 2018;129:583-92.

18. He ZQ, Duan H, Ke C, et al. Evaluation of cumulative prognostic score based on pretreatment plasma fibrinogen and serum albumin levels in patients with newly diagnosed high-grade gliomas. Oncotarget 2017;8:49605-14.
19. He ZQ, Ke C, Al-Nahari F, et al. Low preoperative prognostic nutritional index predicts poor survival in patients with newly diagnosed high-grade gliomas. J Neurooncol 2017;132:239-47.

20. Eckel-Passow JE, Lachance DH, Molinaro AM, et al. Glioma Groups Based on 1p/19q, IDH, and TERT Promoter Mutations in Tumors. N Engl J Med 2015;372:2499-508.

21. Ferrer-Luna R, Mata M, Nunez L, et al. Loss of heterozygosity at $1 \mathrm{p}-19 \mathrm{q}$ induces a global change in oligodendroglial tumor gene expression. J Neurooncol 2009;95:343-54.

22. Hanahan D, Weinberg Robert A. Hallmarks of Cancer: The Next Generation. Cell 2011;144:646-74.

23. Louveau A, Smirnov I, Keyes TJ, et al. Structural and functional features of central nervous system lymphatic vessels. Nature 2015;523:337-41.

24. Chongsathidkiet P, Jackson C, Koyama S, et al. Sequestration of $\mathrm{T}$ cells in bone marrow in the setting of glioblastoma and other intracranial tumors. Nat Med 2018;24:1459-68.

25. Jackson CM, Kochel CM, Nirschl CJ, et al. Systemic Tolerance Mediated by Melanoma Brain Tumors Is Reversible by Radiotherapy and Vaccination. Clin Cancer Res 2016;22:1161-72.

26. Gorrini C, Harris IS, Mak TW. Modulation of oxidative stress as an anticancer strategy. at Rev Drug Discov 2013;12:931-47.

27. Arroyo V, Garcia-Martinez R, Salvatella X. Human serum albumin, systemic inflammation, and cirrhosis. J Hepatol 2014;61:396-407.

28. Moriarty-Craige SE, Jones DP. Extracellular thiols and thiol/disulfide redox in metabolism. Annu Rev Nutr 2004;24:481-509.

29. Kinoshita A, Onoda H, Imai N, et al. Comparison of the prognostic value of inflammation-based prognostic scores in patients with hepatocellular carcinoma. Br J Cancer 2012;107:988-93.

30. Han S, Zhang C, Li Q, et al. Tumour-infiltrating CD4(+) and CD8(+) lymphocytes as predictors of clinical outcome in glioma. Br J Cancer 2014;110:2560-8.

31. Jensen HK, Donskov F, Marcussen N, et al. Presence of intratumoral neutrophils is an independent prognostic factor in localized renal cell carcinoma. J Clin Oncol 2009;27:4709-17.

32. Powell DR, Huttenlocher A. Neutrophils in the Tumor Microenvironment. Trends Immunol 2016;37:41-52.

33. Fridlender ZG, Sun J, Kim S, et al. Polarization of tumor- 
associated neutrophil phenotype by TGF-beta: "N1" versus "N2" TAN. Cancer Cell 2009;16:183-94.

34. Galdiero MR, Bonavita E, Barajon I, et al. Tumor associated macrophages and neutrophils in cancer.

Cite this article as: $\mathrm{He} Z \mathrm{Z}$, Duan $\mathrm{H}$, Lin FH, Zhang J, Chen YS, Zhang GH, Guo CC, Ke C, Zhang XH, Chen ZH, Wang J, Chen ZP, Jiang XB, Mou YG. Pretreatment neutrophil-tolymphocyte ratio plus albumin-to-gamma-glutamyl transferase ratio predict the diagnosis of grade III glioma. Ann Transl Med 2019;7(22):623. doi: 10.21037/atm.2019.11.24
Immunobiology 2013;218:1402-10.

35. Uribe-Querol E, Rosales C. Neutrophils in Cancer: Two Sides of the Same Coin. J Immunol Res 2015;2015:983698. 


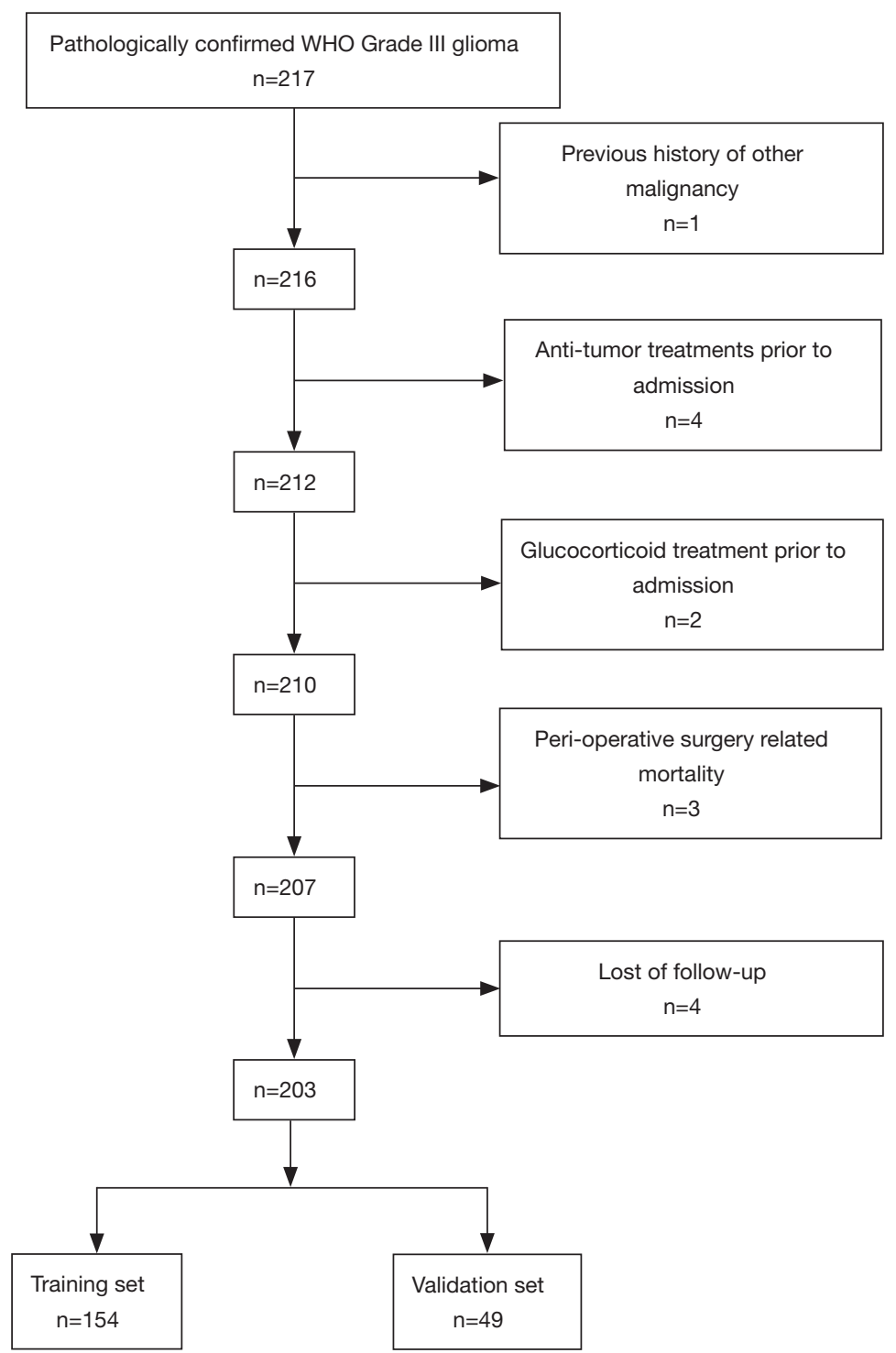

Figure S1 Enrollment flowchart of 217 pathologically confirmed WHO Grade III glioma patients. A total 217 WHO Grade III glioma patients were retrospectively reviewed. One patient was excluded because of previous history of renal carcinoma. Four patients received anti-tumor treatment before admission. Two patients were excluded because of glucocorticoid treatment prior to admission. Three patients were excluded because of perioperative surgery-related mortality. Four patients were lost to follow-up. 203 patients were remained in this cohort. 154 patients treated from January 2001 to July 2014 were enrolled as the training set, and 49 patients identified from August 2014 to December 2016 were enrolled as the validation set. 